\title{
Senescence of Normal Human Fibroblasts Relates to the Expression of Ionotropic Glutamate Receptor GluR6/Grik2
}

\author{
VIKRAMJIT K. ZHAWAR ${ }^{1 \#}$, RAJ P. KANDPAL ${ }^{2 *}$ and RAGHBIR S. ATHWAL ${ }^{1 *}$ \\ ${ }^{1}$ Fels Institute for Cancer Research and Molecular Biology, \\ Temple University School of Medicine, Philadelphia, PA, U.S.A.; \\ ${ }^{2}$ Department of Basic Medical Sciences, Western University of Health Sciences, Pomona, CA, U.S.A.
}

\begin{abstract}
Background/Aim: Glutamate receptor GRIK2, previously designated as GluR6, is best described in neuronal cells. However, its biological relevance in nonneuronal cells is not well understood. We have investigated the expression of this important protein in normal human fibroblasts as a function of cell proliferation. Materials and Methods: We introduced expression constructs of all five isoforms (A-E) of GRIK2 in normal human fibroblasts and investigated the cells for the presence and localization of GRIK2, as well as for cell proliferation and senescence over a period of 24 days. Results: The expression of GRIK2-A isoform led to immediate cessation of cell proliferation. However, the cell numbers increased by 1.5- to 9.0-fold in 24 days upon transfection with B, C, D and E isoforms, after which they entered a state of senescence. The decreased proliferation was reflected by incorporation of BrdU in only 2-8\% of transfected cells even after culturing them for 16 days. Conclusion: Our results are indicative of an association between GRIK2 and aging of fibroblasts.
\end{abstract}

Normal cells proliferate for a finite number of cell divisions before entering replicative senescence and eventually cell death. The interference with this normal cycle can either

This article is freely accessible online.

\#Present address: Department of Biochemistry, Punjab Agricultural University, Ludhiana, 141004, India.

Correspondence to: R.S. Athwal, Fels Institute, Temple University School of Medicine, Philadelphia, PA 19067, U.S.A. Tel: +1 2157076931, e-mail: rathwal@temple.edu and R.P. Kandpal, Department of Basic Medical Sciences, Western University of Health Sciences, Pomona, CA 91766, U.S.A. Tel: +1 9097063520 , e-mail: rkandpal@westernu.edu

Key Words: Senescence, normal fibroblasts, GluR6, Grik2, glutamate receptor, cancer. prolong cellular senescence or prevent cessation of proliferation. Chromosome transfer experiments have led to the identification of various chromosomes that have the ability to induce senescence in carcinoma cell lines (1-4). One such example is mapped to a region encoding the gene for glutamate receptor 6 (GluR6) found on chromosome 6 (5). This gene has been renamed to glutamate receptor ionotropic kainate 2 (GRIK2).

GRIK2 assembles into a multimeric cation channel that is activated by glutamate. GRIK2 can form either homomers or heteromers with other kainate receptor subunits that respond to glutamate (6). We have previously shown that the GRIK2 transcript undergoes alternative splicing to produce five different isoforms (7). These isoforms arise from the action of two promoters and the alternative splicing of the premRNA molecule (7). While the Grik2-A isoform is transcribed from the neuronal promoter, the non-neuronal promoter is activated in epithelial cells to produce other isoforms (8).

To elucidate the molecular basis of senescence induced by chromosome 6 in carcinoma cells, we have previously demonstrated that introduction of engineered Grik2 constructs in carcinoma cells is involved in replicative senescence (9). Furthermore, the transfected carcinoma cells displayed both a phenotype and a molecular signature associated with senescence cells.

Previous results have demonstrated that native GRIK2 isoforms are absent from carcinoma cells and complementation of this defect by transfer of normal chromosome 6 leads to senescence of carcinoma cells (1). These observations have suggested the involvement of GRIK2 as an important factor for defining the life span of cells. In light of these, we investigated Grik2 specific isoform expression in transfected human fibroblasts. Here we present the introduction of specific Grik2 isoforms in fibroblasts, and show their spatial localization and passagedependent expression and relate their overexpression with the cessation of proliferation. 


\section{Materials and Methods}

Cloning of enhanced green fluorescence protein-tagged Grik2 isoforms. We used an insertional mutagenesis protocol to clone 239 codons of the enhanced green fluorescence protein (EGFP) in frame with the Grik2 cDNA after its codon 33 (threonine) in the $p Q C X I P$ vector (Clonetech Laboratories, Mountain View, CA, USA). The first 31 amino acids of Grik2 correspond to its signal peptide. The insertion was accomplished by introducing AgeI and BsrGI restriction sites after codon 33 of Grik2. The full-length open reading frame of EGFP was excised from pBOS-H2GFP (Pharmingen, San Diego, CA, USA) using AgeI and BsrGI restriction sites and was cloned in the Grik2 cDNA digested with the same enzymes (New England Biolabs, Ipswich, MA, USA). The same strategy was applied for cloning the Grik2 transcripts A, B, C, $\mathrm{D}$ and $\mathrm{E}$. Furthermore, a control construct was made by cloning EGFP cDNA only in the same vector.

Retroviral transduction of expression constructs of Grik2 isoforms into normal human dermal fibroblast (HDF) cells. The plasmid DNA corresponding to Grik2 isoforms was isolated and transfected into the HEK293T based Phoenix-amphotropic producer cell line (ATCC, Manassas, VA, USA). The transfected cells were grown for 4-5 days in DME medium (ThermoFisher Scientific, Waltham, MA, USA) with $3 \mu \mathrm{g} / \mathrm{ml}$ puromycin (Sigma-Aldrich, St Louis, MO, USA).

HDF cells (GMO3468) at passage 13 were seeded at a density of $10^{5}$ cells/plate one day before transduction, and puromycin-selected producer phoenix cells were grown at a density of $10^{6} /$ plate. The viral particles-containing medium was aspirated from the producer cells and was passed through a $0.45 \mu \mathrm{m}$ filter (ThermoFisher Scientific) The filtered medium was added to the growing culture of HDF cells. Following $48 \mathrm{~h}$ of transduction, the cells were selected by growing them in the presence of puromycin $(3 \mu \mathrm{g} / \mathrm{ml})$ for three days.

$R T-P C R$. Total RNA was isolated by lysing the cells with Trizol Reagent (Invitrogen/ThermoFisher Scientific) following the manufacturer's protocol. The RNA was precipitated with isopropyl alcohol, the pellet washed with $75 \%$ ethanol and the precipitate dissolved in RNase free water. Approximately 0.5-2 $\mu \mathrm{g}$ of total RNA was converted into cDNA using the Superscript II RT kit (Invitrogen/ThermoFisher Scientific) as per the protocol from the manufacturer. PCR was performed using the protocol and Taq polymerase from Promega (Madison, WI, USA) for 35 cycles of 30 $\mathrm{s}$ at $94^{\circ} \mathrm{C}, 40 \mathrm{~s}$ at $55^{\circ} \mathrm{C}$ and $60 \mathrm{~s}$ at $72^{\circ} \mathrm{C}$ with a final extension of $10 \mathrm{~min}$.

Expression of protein isoforms corresponding to alternatively spliced versions of Grik2 transcript. The transduced cells were fixed onto a slide with $0.2 \%$ glutaraldehyde and $2 \%$ formaldehyde, visualized under an Olympus BX fluorescence microscope (Center Valley, PA, USA), and imaged with a digital camera driven by SPOT software (SPOT Imaging, Sterling Heights, MI, USA).

The transduced cells were lysed using RIPA/PMSF buffer (SigmaAldrich), the lysate was centrifuged and aliquots containing 20-40 $\mu \mathrm{g}$ protein were electrophoresed in an $8 \%$ polyacrylamide gel. The separated proteins were blotted onto PVDF membrane (ThermoFisher Scientific) and probed with anti-GFP (Santa Cruz Biotechnology, Dallas, TX, USA) and anti-GAPDH (Ambion/Thermo Fisher) antibodies.
Proliferation analysis of cells expressing exogenous GRIK2 protein isoforms. The transduced cells were selected in puromycin medium for three days, and were subsequently plated at a density of $10^{4}$ cells/plate in five sets of duplicate plates to assess cell numbers after $2,8,16$, and 24 days. The cell count after $5 \mathrm{~h}$ of plating was considered as the starting number of cells or a zero-time control.

Visualization of senescent cells by assessing inhibition of $\mathrm{BrdU}$ incorporation and the presence of $\beta$-galactosidase activity. Approximately 200-300 transduced cells from 2-day or 16-day cultures were grown in glass bottomed microwell culture dishes (MatTek Corporation, Ashland, MA, USA). After growing the cells for $24 \mathrm{~h}$ in Eagle's medium (ThermoFisher Scientific), the culture medium was supplemented with 5-bromo-deoxy-uridine (SigmaAldrich) to a final concentration of $10 \mu \mathrm{M}$ and cells were further incubated for additional $24 \mathrm{~h}$. The cells were fixed in $4 \%$ paraformaldehyde and were treated with $4 \mathrm{~N} \mathrm{HCl}$. The cells were then washed with $0.1 \mathrm{M}$ sodium borate $(\mathrm{pH} 8.5)$, permeabilized with $0.25 \%$ Triton X-100 (Sigma-Aldrich), and were treated with blocking buffer $(5 \%$ fetal bovine serum from ThermoFisher and $0.25 \%$ Triton X-100 from Sigma-Aldrich in phosphate buffered saline). The cells were incubated overnight with a mouse anti-BrdU antibody (Molecular Probes/Thermo-Fisher Scientific). The slides were subsequently incubated with Texas Red-labeled anti-mouseIgG antibody (Jackson ImmunoResearch Labratories, West Grove, PA, USA). The cells were visualized under an Olympus BX fluorescence microscope (Olympus, Center Valley, PA, USA) and imaged using a SPOT software-driven digital camera (SPOT Imaging). The incorporation of $\mathrm{BrdU}$ is a measure of DNA replication and cell proliferation.

To evaluate senescence, cells from 2-day and 16-day cultures were fixed with a solution of $2 \%$ formaldehyde and $0.2 \%$ glutaraldehyde in PBS. After $24 \mathrm{~h}$, the cells were incubated with XGal reagent $(1 \mathrm{mg} / \mathrm{ml}$, ThermoFisher Scientific) in $40 \mathrm{mM}$ sodium citrate buffer ( $\mathrm{pH}$ 6), $5 \mathrm{mM}$ potassium ferrocyanide, $5 \mathrm{mM}$ potassium ferricyanide, $150 \mu \mathrm{M} \mathrm{NaCl}$ and $2 \mathrm{mM} \mathrm{MgCl}_{2}$ for $12 \mathrm{~h}$ at $37^{\circ} \mathrm{C}$ in the dark. Thereafter, cells were imaged using the Olympus phase-contrast microscope mentioned above attached to a SPOT digital camera.

Immunofluorescence for surface expression of Grik2 protein isoforms. The cells selected in puromycin-containing medium were grown in glass bottom microwell dishes (MatTek Corporation, Ashland, MA, USA) for one day and subsequently treated with mouse anti-GFP monoclonal antibody (Santa Cruz Biotechnology) for one $\mathrm{h}$. Next, the cells were fixed with $4 \%$ paraformaldehyde, incubated in blocking buffer (1X PBS, 5\% serum and $0.3 \%$ Triton $\mathrm{X}-100$ ) and treated with anti-mouse IgG-Texas Red (Jackson ImmunoResearch Laboratories) for one $\mathrm{h}$ at room temperature. The cells were washed with PBS, and were imaged by a SPOT digital camera attached to the Olympus BX fluorescence microscope for green and red fluorescence as described before. The relative intensity of red fluorescence on the cell surface to the total EGFP was determined by imaging the cells at a constant gain and exposure-time in the AlphaImager system using the AlphaEase ${ }^{\mathrm{TM}}$ software (Alpha Innotech Corporation, San Jose, CA, USA). It is noteworthy that while the red fluorescence due to Texas red conjugated anti-mouse IgG would detect EGFP-tagged Grik2 protein that traffics to the cell surface, green fluorescence would correspond to total Grik2 abundance. 


\section{Results}

Grik2 Abundance in early and late passage HDF cells. To check whether there is an age-dependent expression of Grik2 in HDF cells, we harvested cells after 18 (early) and 70 (late) passages. Total RNA was isolated from these cells and two representative isoforms ( $\mathrm{B}$ and $\mathrm{D})$ were reverse transcribed and subsequently amplified by PCR. The levels of these transcripts were compared to the abundance of GAPDH. It is clear that while the abundance of GAPDH is nearly comparable in both early and late passage, the levels of Grik2-B and D isoforms are significantly higher in the late passage cells compared to the early passage cells (Figure 1).

Expression of Grik2 protein isoforms in HDF cells. The five isoforms of Grik2 protein can be distinguished by the presence or absence of transmembrane domains and the $\mathrm{C}$ terminus ends. The identification of native isoforms by western blotting and cell imaging was not feasible due to lack of isoform-specific antibodies. We, therefore, transduced fibroblasts with engineered EGFP-tagged retroviral constructs containing these isoforms. The fluorescence of EGFP in transduced cells was indicative of the expression of Grik2 isoforms in the transduced cells. As shown in Figure 2A, EGFP by itself shows uniform expression throughout the cell, including the nucleus. However, a significant proportion of $\mathrm{A}, \mathrm{B}, \mathrm{C}, \mathrm{D}$ and $\mathrm{E}$ isoforms displayed non-nuclear localization.

The confirmation of actual isoforms was demonstrated by western blotting of the lysates from cell transduced with specific isoforms. As shown in Figure 2B, the anti-EGFP antibody recognized specific size protein bands that correspond to predicted molecular weight of EGFP-tagged GRIK2 isoforms. We could not detect isoform-A because the number of cells transduced with this isoform was too small to isolate sufficient amount of protein for detection by blotting. The cells transduced with the EGFP construct by itself showed a protein band of $\sim 27 \mathrm{kD}$, which corresponds to the predicted size of EGFP. These results clearly demonstrate the expression of expected sizes of transduced GRIK2 isoforms in the transfected fibroblasts (8).

Grik2 isoforms suppress the growth of fibroblasts. The proliferation of cells transduced with Grik2 isoforms was determined by counting the cells after 2, 8, 16 and 24 days of growth. As indicated in Figure 3, the proliferation of EGFP-expressing cells was not significantly different from the parent cell line GMO3468. However, expression of the Grik2 isoforms in native cells consistently inhibited their proliferation. The isoform A, in particular, prevented proliferation as indicated by the nearly static number of cells even after 24 days in the growth medium. The other Grik2 isoforms differentially inhibited cell proliferation. The count

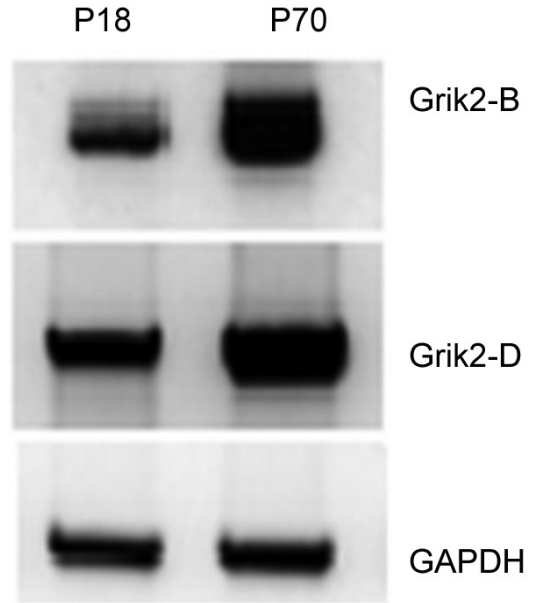

Figure 1. Abundance of Grik2 isoforms $B$ and $D$ in human dermal fibroblasts. Total RNA was isolated from human dermal fibroblasts (HDF) at passages $18(P 18)$ and P70, respectively. The abundance of the two transcripts was determined by RT-PCR using isoform-specific primers. GAPDH primers were used to determine the passage-specific relative abundance of Grik2.

for cells expressing isoforms $\mathrm{B}$ and $\mathrm{C}$ increased marginally from $10^{4}$ to $1.5 \times 10^{4}$ and $1.8 \times 10^{4}$, respectively, over a period of 24 days. The inhibitory effects of isoform-D and isoformE were the lowest as compared to the other variants. The cells expressing these isoforms increased from $10^{4}$ to $9 \times 10^{4}$ and $5 \times 10^{4}$, respectively, in 24 days.

Grik2 isoforms induce inhibition of DNA replication. To evaluate the effects of Grik2 isoforms on DNA replication, the proliferating and non-proliferating cells were distinguished using the BrdU incorporation assay. After growing the cells in the presence of BrdU at days 2 and 16, the cell count was determined by staining the cells with a fluorescent anti-BrdU antibody. As shown on day 2 in Figure $4 \mathrm{~A}$, while more than $90 \%$ of the control vector expressing cells had BrdU incorporated in them, the portion of positive cells varied between $10-30 \%$ in the presence of the various Grik2 isoforms. The lowest number of BrdU-positive cells were observed in those expressing the isoform-A. Although the incorporation in control cells at day 16 decreased marginally, a significant decrease was observed in the presence of the other isoforms of Grik2. The quantitation of cells indicated that BrdU incorporation occurred in only 2$8 \%$ of cells transduced with Grik2, demonstrating a correlation between the decrease in proliferation and the inhibition of DNA replication in the presence of the various isoforms of Grik2. A representative image of stained cells in the presence of control vector or a Grik2 isoform is shown in Figure 4B. 
A
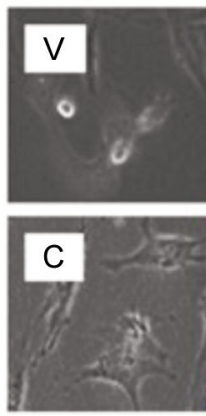
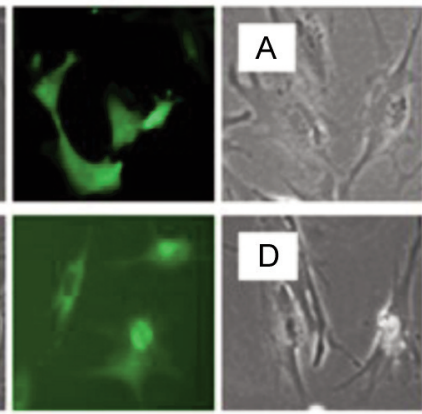
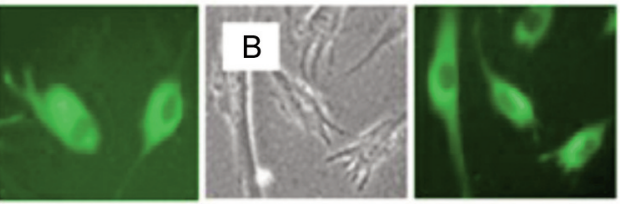

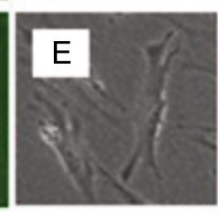

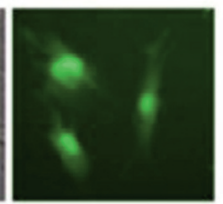

B

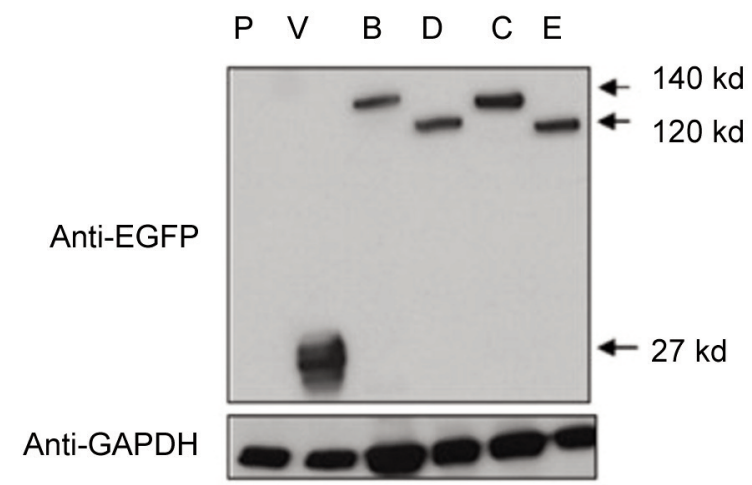

Figure 2. Expression of EGFP-tagged Grik2 isoforms in transfected human dermal fibroblasts. A) Images of cells under a phase-contrast and fluorescence microscope. The phase-contrast and florescent images of cells transduced with the vector or isoforms A-E of Grik2, labeled as $V$, A, $B, C, D$ and E. B) Proteins isolated from native cells $(P)$ and cells transduced with the vector (V) or constructs of Grik2 isoforms B-E were probed with an anti-EGFP antibody (upper panel). The total protein was quantified by probing the blot with an anti-GAPDH antibody (lower panel). Images were acquired at $100 \times$ magnification.

Grik2 isoforms induce senescence in fibroblasts. The replicative senescence in transduced cells was investigated by assessing the morphology and distinct biochemical phenotype of cells expressing the various isoforms of Grik2. We evaluated these cells for senescence-associated $\beta$ galactosidase activity on days 2 and 16 . The number of bluecolored cells ( $\beta$-galactosidase-positive) was determined as a fraction of total cells at three different locations in the plate. As shown in Figure 5A, less than $10 \%$ of cells transduced with the control vector stained blue on day 2 , whereas the portion of $\beta$-galactosidase-positive cells varied between $45 \%$ and $60 \%$ in the presence of various isoforms of Grik2. The abundance of $\beta$-galactosidase-positive cells increased to up to $95 \%$ on day 16 , as compared to $\sim 20 \%$ in the vector control. Figure $5 \mathrm{~B}$ shows a representative field of stained cells. These results demonstrate the induction of senescence in cells that express the various isoforms of Grik2.

Trafficking of Grik2 isoforms to fibroblast cell surface. The isoform-D and isoform-E lack three of the five exons coding for the transmembrane domains. It was therefore pertinent to investigate whether these isoforms are transported to cell surface, using the EGFP reporter. As described in the methods section, Texas red tags the anti-mouse IgG and recognizes the cell surface-localized Grik2 while green fluorescence indicates total Grik2. Thus, the differential intensity of green and red fluorescence is suggestive of the variable trafficking of different Grik2 isoforms. Figure 6 shows the signal corresponding to EGFP throughout the cell and on the surface specifically. It is evident from the images that isoform-A trafficking occurred most efficiently, even though all other isoforms also moved to the cell surface with reasonable efficiency, apart from isoform E. The lack of three transmembrane domain exons in isoform $\mathrm{D}$ does not appear to compromise its ability to localize onto the cell surface, while the same cannot be said for isoform $\mathrm{E}$.

\section{Discussion}

The mechanisms of genomic instability and metabolic stress due to aging have been investigated extensively (10-12). These hypotheses postulate that mutations accumulate over 


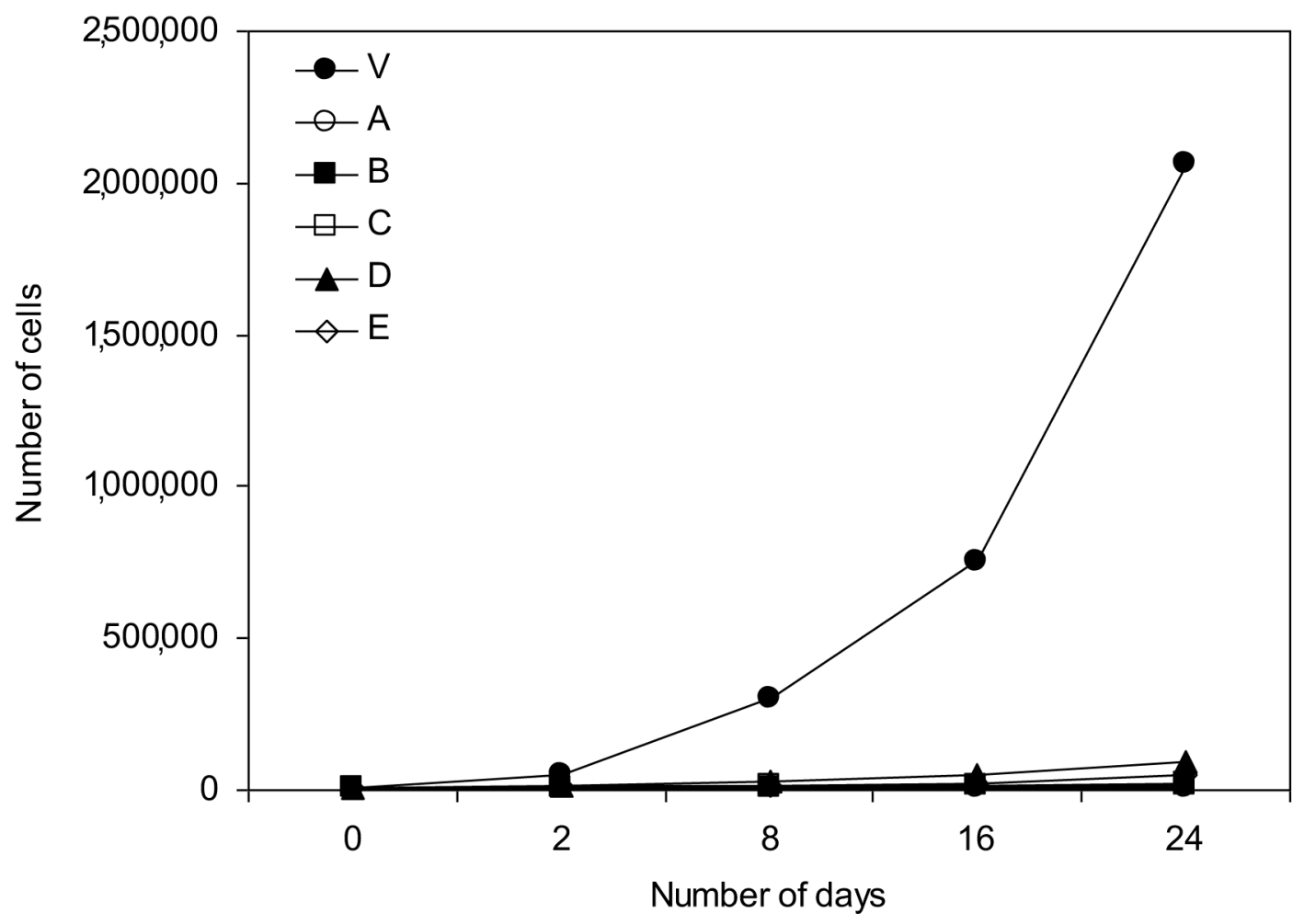

Figure 3. Proliferation of Grik2-transducedd human dermal fibroblasts. Cells were transduced either with the vector (V) or with constructs corresponding to Grik2 isoforms A-E. The zero day indicates the number of cells on day 3 after transduction. The values represent averages of cell counts for two experiments.

a lifetime, impairing stress responses and causing inflammatory pathways eventually culminate into cellular injuries that are associated with aging (13-16). Telomere shortening due to end-replication issues has been proposed as the basis of the Hayflick limit on cell divisions before cells enter replicative senescence (17). Although numerous molecular changes have been attributed to senescence and cancer phenotypes, our understanding of aging mechanisms remains far from complete (18). We have explored a novel strategy to identify molecular determinants of senescence, which involves transfer of normal human chromosomes into immortalized cell lines (1). This strategy has led to the isolation of Grik2 as a senescence-causing gene (9). We have demonstrated here that the senescence-inducing activity in fibroblasts is associated with various isoforms of Grik2. Our conclusions are drawn based on the effects of these isoforms on the cell doubling time as well as the expression of senescence-associated $\beta$-galactosidase activity in the transfected human fibroblasts.

We have previously described the expression of five isoforms of Grik2, which result from the activation of two tissue-specific promoters and alternative splicing of precursor mRNA (7). It is noteworthy that Grik2-A is abundant in neuronal cells (19), and forced expression of this isoform in fibroblasts completely blocks their proliferation and replication; however, the cells remain alive in the medium without undergoing programmed cell death. The senescence of these cells is evident by their morphology and abundant expression of senescence-associated $\beta$ galactosidase activity. It is not surprising that the neuronal isoform of Grik2 induces the most robust senescence phenotype in fibroblast cells. The senescence-associated features, such as heterochromatin formation and inflammatory cytokines have been reported in neuronal cells (20), and these cells also show elevated activity of $\beta$ galactosidase. The absence of the neuronal isoform in normal non-neuronal cells can be attributed to transcriptional silencing of the neuronal promoter by epigenetic mechanisms (21). Thus, the expression of the A-isoform may serve a dual purpose of maintaining the differentiated status as well as regulating the action potential of neuronal cells.

We have observed progressive increase in the expression of Grik2 transcripts at late passages in fibroblast cells. The specific expression of isoforms B-E is due to the activation of the alternative promoter and alternative splicing mechanisms operating in these cells. The engineered 


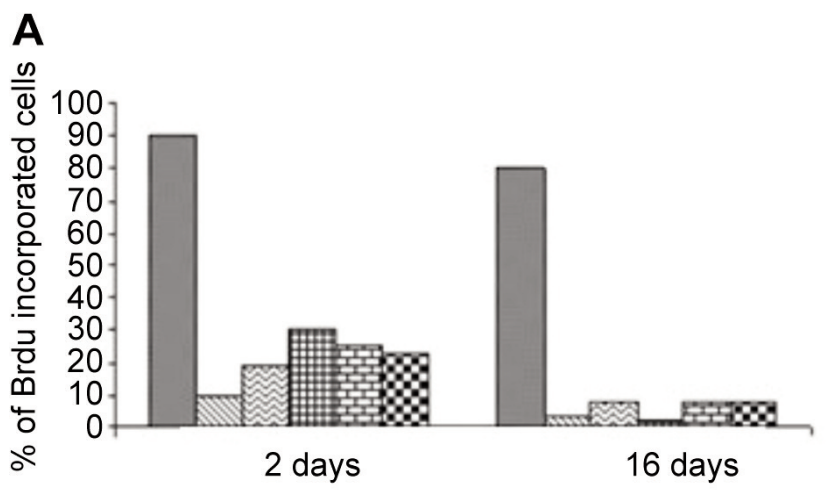

B

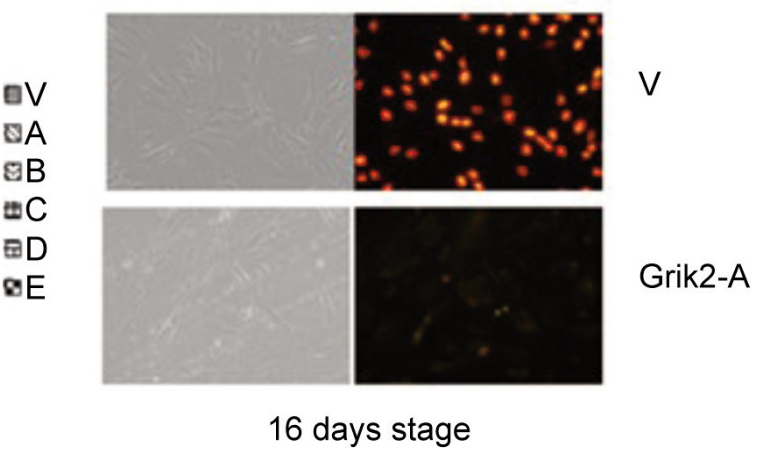

Figure 4. Incorporation of BrdU in Grik2-transduced human dermal fibroblasts. A) Cells were grown in the presence of BrdU. The bars indicate the percentage of cells showing BrdU incorporation in cells transduced with the vector ( $V$ ) or isoforms A-E of Grik2 on days 2 and 16 . The numbers represent averages of counts from three fields of view. B) Phase-contrast or fluorescence microscopy of cells transduced with vector (V) or a Grik 2 construct. The images were acquired at $100 \times$ magnification.

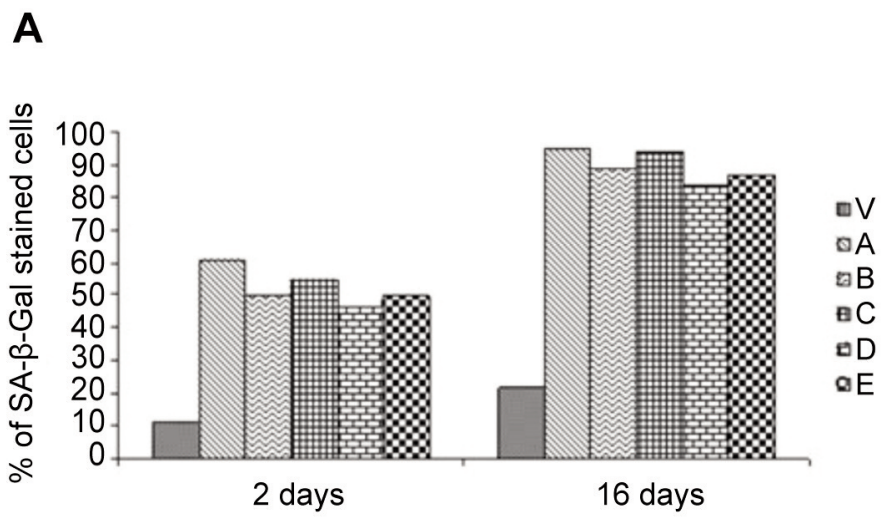

Figure 5. Quantification and detection of senescent human dermal fibroblasts. A) The cells transduced with vector (V) and isoforms A-E of Grik2 were grown in the presence of the $X$-Gal reagent to detect senescence-associated $\beta$-galactosidase (SA $\beta$-gal). The bars indicate the number of stained cells, and represent averages of counts from three fields of view. B) The representative field views of stained cells transduced with the vector (V) and Grik2 isoforms A-E. Images were acquired at 100x magnification.

expression of these isoforms and the consequent senescence were confirmed by i) enhanced senescence-associated $\beta$ galactosidase activity, ii) inhibition of BrdU incorporation and iii) surface localization of the Grik2 isoforms in the transduced cells indirectly through Texas red/EGFP expression. These results are indicative of Grik2 being involved in inducing replicative senescence. It is noteworthy that more than $90 \%$ cells exhibited senescence-associated $\beta$ galactosidase activity at day 24 . We reason that basal expression of these isoforms continues in normal cells, and speculate some involvement of epigenetic mechanisms in stabilizing the senescent state in cells at late passages.

\section{B}

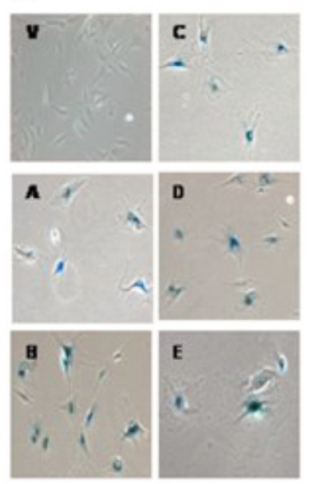

16 days 


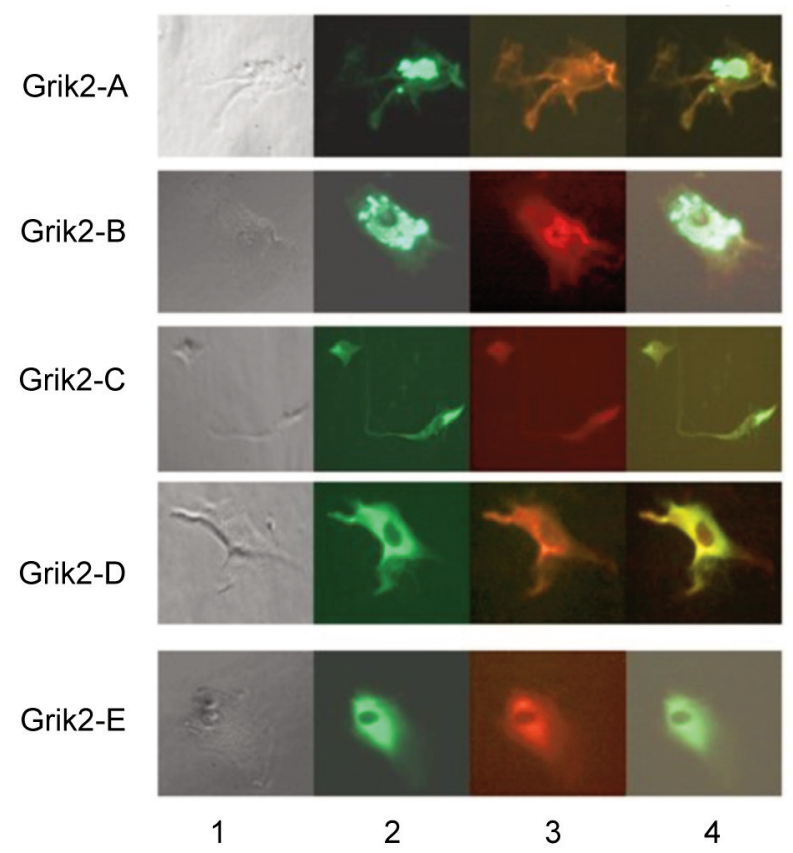

Figure 6. Trafficking of Grik2 to the cell surface of human dermal fibroblasts. The cells were transduced with isoforms A-E, and the total protein and surface expression were detected. The images were obtained by phase contrast (Panel 1), total expression by EGFP fluorescence (Panel 2), surface expression by Texas red fluorescence (Panel 3), and differential localization by merging the two fluorescent channels (Panel 4). Images were acquired at 100x magnification.

speculate that the channels formed by these isoforms do not allow the passage of ions. We believe that, upon binding to their ligands, these receptors participate in signaling pathways and transduce conformational changes to allow interactions with other intracellular proteins (23). Similar biological significance may be proposed for the $\mathrm{B}$ and $\mathrm{C}$ isoforms.

We have previously shown that transfer of native Grik2 constructs in ovarian carcinoma cells prevents their proliferation and induces a senescent phenotype (9). Thus, the dual role of this protein is plausible. While in neuronal cells the channel conformation of the protein primarily mediates action potential, the cytosolic domain may undergo a conformational change to maintain the differentiated status of the neuron. This proposition would fit well with noncanonical functionality of ionotropic receptors (23). The latter function, which includes senescence induction, may likely be controlled by a pathway common to all isoforms. Therefore, we hypothesize genetic and epigenetic mutations in Grik2 as important aspects of tumorigenesis in some human cancers. Our studies suggest a yet to be determined role of Grik2 in normal aging, and implicate mutants of these proteins in the possible escape from senescence in cancer.
The downstream effectors of Grik2 on senescence may also be relevant for age-related diseases. Some important genes, such as p53 and p16 Ink4a possibly function to link senescence with complex biological processes, such as aging (22). It is noteworthy that while senescence induction would be desirable for carcinoma cells, a context-dependent senescence-associated secretory phenotype has been implicated in tumor promoting inflammatory responses (22). In conclusion, our studies tentatively support a novel cellular phenotype associated with Grik2, which may have important implications in aging and cancer.

\section{Conflicts of Interest}

The Authors declare no conflicts of interest.

\section{Authors' Contributions}

VKZ performed experiments and data analysis, RSA directed the overall project, and RPK participated in experimental design and wrote the manuscript draft.

\section{Acknowledgements}

The Authors wish to acknowledge the shared resources provided by Fels Institute.

\section{References}

1 Kandpal RP, Sandhu AK, Kaur G, Kaur GP and Athwal RS: Monochromosomal hybrids and chromosome transfer: A functional approach for gene identification. Cancer Genomics Proteomics 14(2): 93-101, 2017. PMID: 28387649. DOI: $10.21873 /$ cgp.20022

2 Sandhu AK, Hubbard K, Kaur GP, Jha KK, Ozer HL and Athwal RS: Senescence of immortal human fibroblasts by the introduction of normal human chromosome 6. Proc Natl Acad Sci USA 91(12): 5498-5502, 1994. PMID: 8202516. DOI: 10.1073/pnas.91.12.5498

3 Banga SS, Kim S, Hubbard K, Dasgupta T, Jha KK, Patsalis P, Hauptschein R, Gamberi B, Dalla-Favera R, Kraemer P and Ozer HL: SEN6, a locus for SV40-mediated immortalization of human cells, maps to 6q26-27. Oncogene 14(3): 313-321, 1997. PMID: 9018117. DOI: 10.1038/sj.onc.1200842

4 Acquati F, Morelli C, Cinquetti R, Bianchi MG, Porrini D, Varesco L, Gismondi V, Rocchetti R, Talevi S, Possati L, Magnanini C, Tibiletti MG, Bernasconi B, Daidone MG, Shridhar V, Smith DI, Negrini M, Barbanti-Brodano G and Taramelli R: Cloning and characterization of a senescence inducing and class II tumor suppressor gene in ovarian carcinoma at chromosome region 6q27. Oncogene 20(8): 980988, 2001. PMID: 11314033. DOI: 10.1038/sj.onc.1204178

5 Rane NS, Sandhu AK, Zhawar VS, Kaur G, Popescu NC, Kandpal RP, Jhanwar-Uniyal M and Athwal RS: Restoration of senescence in breast and ovarian cancer cells following the transfer of the YAC carrying SEN6A gene located at 6q16.3. Cancer Genomics Proteomics 8(5): 227-233, 2011. PMID: 30587499 . 
6 Contractor A, Mulle C and Swanson G: Kainate receptors come of age: milestones of two decades of research. Trends Neurosci 34(3): 154-163, 2011. PMID: 21256604. DOI: 10.1016/j.tins.2010.12.002

7 Barbon A, Vallini I and Barlati S: Genomic organization of the human GRIK2 gene and evidence for multiple splicing variants. Gene 274(1-2): 187-197, 2001. PMID: 11675011. DOI: 10.1016/s0378-1119(01)00611-4

8 Zhawar VK, Kaur G, deRiel JK, Kaur GP, Kandpal RP and Athwal RS: Novel spliced variants of ionotropic glutamate receptor GluR6 in normal human fibroblast and brain cells are transcribed by tissue specific promoters. Gene 459(1-2): 1-10, 2010. PMID: 20230879. DOI: 10.1016/j.gene.2010.03.002

9 Zhawar VK, Kandpal RP and Athwal RS: Isoforms of ionotropic glutamate receptor GRIK2 induce senescence of carcinoma cells. Cancer Genomics Proteomics 16(1): 59-64, 2019. PMID: 30587499. DOI: $10.21873 / \operatorname{cgp} .20111$

$10 \mathrm{Vijg} \mathrm{J}$ and Suh Y: Genome instability and aging. Annu Rev Physiol 75: 645-668, 2013. PMID: 23398157. DOI: 10.1146/annurev-physiol-030212-183715

11 Kirkwood TB: Understanding the odd science of aging. Cell 120(4): 437-447, 2005. PMID: 15734677. DOI: 10.1016/ j.cell.2005.01.027

12 Kenyon CJ: The genetics of ageing. Nature 464(7288): 504-512, 2010. PMID: 20336132. DOI: $10.1038 /$ nature08980

13 Kirkwood TB and Proctor CJ: Somatic mutations and ageing in silico. Mech Ageing Dev 124(1): 85-92, 2003. PMID: 12618010. DOI: $10.1016 / \mathrm{s} 0047-6374(02) 00177-\mathrm{x}$

14 Kriete A, Bosl WJ and Booker G: Rule-based cell systems model of aging using feedback loop motifs mediated by stress responses. PLoS Comput Biol 6(6): e1000820, 2010. PMID: 20585546. DOI: 10.1371/journal.pcbi.1000820

15 Saunders LR and Verdin E: Stress response and aging. Science 323(5917): 1021-1022, 2009. PMID: 19229027. DOI: 10.1126/ science. 1170007
16 Campisi J: Aging, cellular senescence, and cancer. Annu Rev Physiol 75: 685-705, 2013. PMID: 23140366. DOI: 10.1146/annurev-physiol-030212-183653

17 Rodriguez-Brenes IA and Peskin CS: Quantitative theory of telomere length regulation and cellular senescence. Proc Natl Acad Sci USA 107(12): 5387-5392, 2010. PMID: 20207949. DOI: $10.1073 /$ pnas.0914502107

18 Wiley CD and Campisi J: From ancient pathways to aging cellsconnecting metabolism and cellular senescence. Cell Metab 23(6): 1013-1021, 2016. PMID: 27304503. DOI: 10.1016/ j.cmet.2016.05.010

19 Coussen F, Perrais D, Jaskolski F, Sachidhanandam S, Normand E, Bockaert J, Marin P and Mulle C: Co-assembly of two GluR6 kainate receptor splice variants within a functional protein complex. Neuron 47(4): 555-566, 2005. PMID: 16102538 . DOI: $10.1016 /$ j.neuron. 20056.06 .033

20 Tan FC, Hutchison ER, Eitan E and Mattson MP: Are there roles for brain cell senescence in aging and neurodegenerative disorders? Biogerontology 15(6): 643-660, 2014. PMID: 25305051. DOI: $10.1007 / \mathrm{s} 10522-014-9532-1$

21 Sen P, Shah PP, Nativio R and Berger SL: Epigenetic mechanisms of longevity and aging. Cell 166(4): 822-839, 2016. PMID: 27518561. DOI: 10.1016/j.cell.2016.07.050

22 Rodier F and Campisi J: Four faces of cellular senescence. J Cell Biol 192(4): 547-556, 2011. PMID: 21321098. DOI: 10.1083/ jcb.201009094

23 Valbuena S and Lerma J: Non-canonical signaling, the hidden life of ligand-gated ion channels. Neuron 92(2): 316-329, 2016. PMID: 27764665. DOI: 10.1016/j.neuron.2016.10.016

Received August 26, 2020

Revised September 10, 2020

Accepted September 11, 2020 